\title{
Histopathological Study and Expression of CD 117 in Renal Cell Carcinoma
}

\author{
Rasmi Unnikrishnan ${ }^{1 *}$, Praseeda I ${ }^{2}$ and Santha Sadashivan ${ }^{1}$ \\ ${ }^{1}$ Department of Pathology, Sree Narayana Institute of Medical Sciences, Ernamkulam, Kerala, India \\ ${ }^{2}$ Department of Pathology, Travencore Medical College, Kollam, Kerala, India
}

\section{ABSTRACT}

Background: Hypernephroma or Renal Cell Carcinoma (RCC) accounts for over $90 \%$ of all malignancies of the kidney occurring in adults. Of all renal cell carcinomas, Clear cell carcinoma accounts for around two-thirds to three-quarters andChromophobe RCC accounts for around 5\%. The present study highlights the diagnostic usefulness of CD117 as a phenotypic marker in Renal Cell Carcinoma.

Methods: Current study include 48 cases of histopathology proven renal cell carcinoma. Each case was reviewed and classified according to the WHO classification criteria. The expression of CD117 was assessed using immunohistochemistry.

Result: Of the 48 cases studied, 6cases, that is 2 case of Papillary RCC and 3 case of Chromophobe RCC showed moderate intensity and diffuse positivity for CD 117 and 1 case of clear cell RCC with sarcomatoid areas showed mild and focal positivity. Papillary RCC showed both cytoplasmic and membrane positivity, while Chromophobe RCC showed mainly membranous positivity. Sarcomatoid areas showed weak cytoplasmic positivity.

Conclusion: Histopathology supported by immunohistochemistry is a helpful tool in subtyping the various RCC. Treatment options in advanced disease is minimal. CD 117 positivity may favour the use of targeted therapy in renal cell carcinomas.

\section{Keywords: Renal Cell Carcinoma, Histopathological Study, Immunohistochemistry}

\section{Introduction}

Renal cell carcinoma (RCC) is the third most common urological malignancy following prostate and urinary bladder malignancies and accounts for $3 \%$ of all malignancies ${ }^{[1]}$. It is the seventh most common cancer in men and the ninth most common in women. It generally affects adults of average age 55-60years. Its incidence increases with each decade of life until the sixth decade and it is two to three times more common in males than in females ${ }^{[2]}$. With modern imaging techniques, such as ultrasound (US) and computerised tomography (CT), the number of incidentally diagnosed RCCs has increased ${ }^{[3,4]}$.

Renal cell carcinoma represents a heterogeneous group of tumors with potential prognostic and therapeutic differences. Hence in the majority of cases, reaching a final definite diagnosis is possible by examining the tumor's gross and light microscopic morphologic features. However, there is a sufficient overlap between several entities and moreover in case of small biopsies where the entire range of cytoarchitectural features may not be fully appreciated the need for ancillary techniques comes into play in order to narrow down the differential diagnosis or to arrive at a definitive diagnosis ${ }^{[5,6]}$. The treatment options in Renal Cell Carcinoma depends on the stage of disease and mainly includes partial / radical nephrectomy. Around $20-30 \%$ of patients undergoing nephrectomy experience relapse and develop metastasis ${ }^{[7,8]}$. Newer modalities include targeted therapy with VEGF inhibitors. Currently we do not have any effective treatment for metastatic disease apart from surgical procedures. Overexpression of CD 117 on cellular membranes of tumor cells could be a potential target for kinase inhibitors. The aim of my study is to histologically study RCC and to look for expression of CD117 in the tumor ${ }^{[9,10]}$.

\section{Materials and Methods}

This is a descriptive study done in all nephrectomy specimens of histopathology proven renal cell carcinoma received in a major tertiary hospital in South Kerala, India, during the period from July 2013 to June 2014 which was given clearance by the Institutional Ethics Committee. Clinical details and staging of all cases of renal cell carcinoma were collected. Formalin fixed paraffin embedded sections were cut into $5 \mu \mathrm{m}$-thick sections and were stained with $\mathrm{H}$ $\&$ E. Each case was reviewed and classified according to the WHO classification criteria. The expression of CD117 was assessed using immunohistochemistry. The staining condition was adjusted using GIST samples known to express c-KIT as positive controls. The tissue mast cells, which stain $3+$, also served as a positive control. Negative 
controls included omitting the primary antibody. The c-KIT positive cases were evaluated using an evaluation system for c-KIT immunostaining proposed by Miliaras, et al. It included evaluation of the intensity as well as the extent of staining. All results and data were entered in Excel sheet and analysed by using appropriate statistical software SPSS.

\section{Result}

Among the 48 cases studied, the age of patients ranged from 30 to 80 yrs with a mean age of 55.39 and a median age of 58yrs. Of these, 36 (75 percent) were males and 12 (25 percent) were females. Thus the male to female ratio was $3: 1$, showing a definite male preponderance.

At least one of the classic symptoms of renal cell carcinoma ie., palpable mass, hematuria or abdominal pain was present in 25 (52\% of the patients) cases. Incidentally detected patients constitute around $23(48 \%)$ cases. Hematuria was the most common presenting symptom which was found in 25 patients. Around 6 cases showed the classical triad of Renal Cell Carcinoma.

Out of the 48 specimens received, 37 were that of radical nephrectomy (77.1\%) and the remaining 11 (22.9\%) were of partial nephrectomy. Left kidney was the biopsy specimen in 27 cases $(56.25 \%)$. On gross examination most of the tumors were of the size $<7 \mathrm{~cm}$, that is 32 out of 48 patients $(66.67 \%)$ belonged to $\mathrm{T} 1$ group. Rest of the 16 cases had tumor size $>7 \mathrm{~cm}$.
The most common histology was Clear cell type, with variable cytoplasmic features ranging from clear cell in 30 cases $(62.5 \%)$ to mixed clear and oncocytic morphology in 2 cases. Five cases of Clear cell RCC showed sarcomatoid change in the form of pleomorphic sarcomatous areas constituting $10.42 \%$ of cases. Apart from these, Clear cells with Papillary areas were seen in 1 case. Papillary RCC (Fig:3) and Chromophobe RCC (Fig:1) constituted around $6.25 \%$ ( 3 cases) each. Other less common types of RCC includes, Unclassified RCC - 4.17\% (2 cases), Multilocular cystic RCC - 2.08\% ( 1 case), and Mucinous Tubular and Spindle Cell RCC - 2.08\% ( 1 case).

Of the 48 cases studied, 6 cases, that is 3 case of Chromophobe RCC and 2 case of Papillary RCC showed moderate intensity and diffuse positivity for CD 117 and 1 case of clear cell RCC with sarcomatoid areas showed mild and focal positivity. Papillary RCC showed both cytoplasmic and membrane positivity (Fig: 4), while Chromophobe RCC showed mainly membranous positivity (Fig: 2). Sarcomatoid areas showed weak cytoplasmic positivity (Fig: 5). While all other cases were negative for CD 117. The positive cases were scored according to the intensity and extend of positivity used by Miliaras, et al (Table: 1). Accordingly, c-KIT immunoreactive cases of scores $\geq 4$ were considered positive, whereas, those below score 4 were considered negative. One case of Papillary RCC with a score of 4 and another case of Papillary RCC and all the 3 cases of Chromophobe RCC with a score of 5 each were considered positive.

Table:1: Scoring of cases positive for CD 117.

\begin{tabular}{|c|c|c|c|c|}
\hline & $\begin{array}{l}\text { N.o positive cases / } \\
\text { N.o total cases }\end{array}$ & Intensity & Extent & Score \\
\hline Clear Cell RCC & $0 / 30$ & 0 & _ & _- \\
\hline - With Oncocytic Change & $0 / 2$ & 0 & 0 & 0 \\
\hline - $\quad$ With Sarcomatoid Change & $1 / 5$ & 1 & 1 & 2 \\
\hline - With Papillary Areas & $0 / 1$ & 0 & 0 & 0 \\
\hline Papillary RCC & $2 / 3$ & & & \\
\hline $\begin{array}{l}\text { Positive- } \\
-\quad \text { Case } 1\end{array}$ & & 1 & 3 & 4 \\
\hline - $\quad$ Case 2 & & 2 & 3 & 5 \\
\hline Chromophobe RCC & $3 / 3$ & 2 & 3 & 5 \\
\hline Multilocular Cystic RCC & $0 / 1$ & 0 & _ & _ \\
\hline Mucinous Tubular And Spindle Cell RCC & $0 / 1$ & 0 & . & . \\
\hline Unclassified RCC & $0 / 2$ & 0 & - & _- \\
\hline
\end{tabular}

Table 2: Comparing CD117 positivity in different studies:

\begin{tabular}{|c|c|c|c|c|}
\hline & N.o of positive cases & Clear cell RCC & Chromophobe RCC & Papillary RCC \\
\hline Present study & $6 / 48$ & 1 & $\mathbf{3}$ & $\mathbf{2}$ \\
\hline Eman AA et al & $14 / 49$ & $\mathbf{4}$ & $\mathbf{7}$ & $\mathbf{0}$ \\
\hline Miliaras et al & $8 / 25$ & $\mathbf{2}$ & $\mathbf{4}$ & $\mathbf{2}$ \\
\hline
\end{tabular}




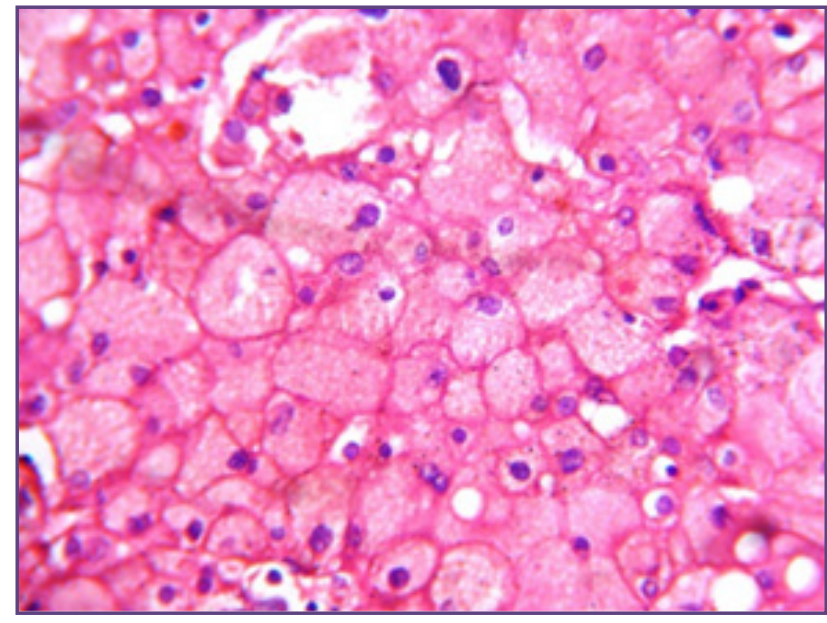

Fig. 1: H \& E (40x) Chromophobe RCC.

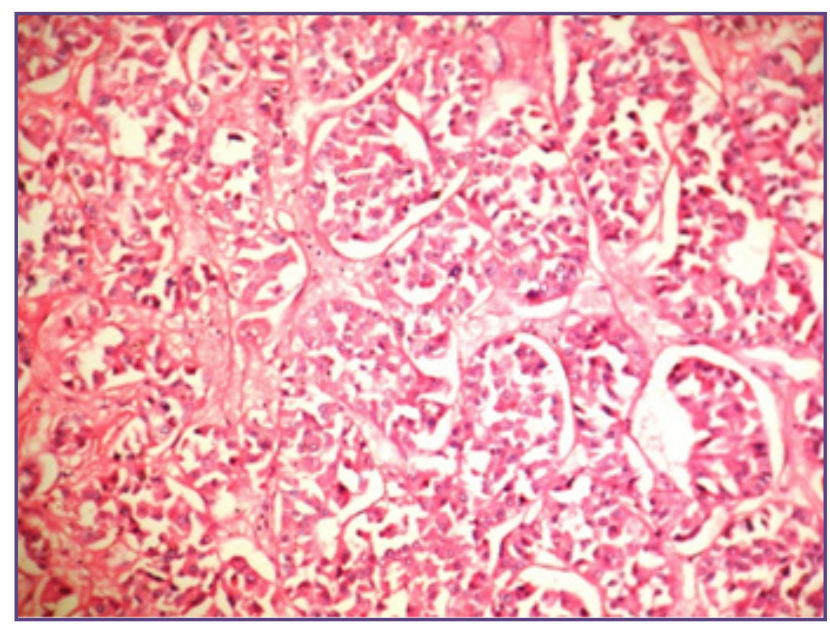

Fig. 3: H \& E (40x) Papillary RCC.

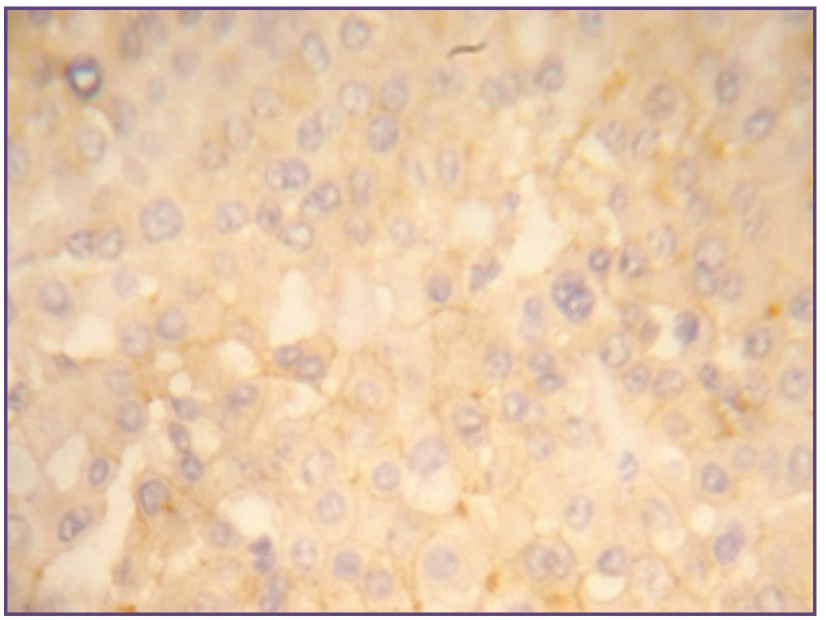

Fig. 2: CD117 (40x) Cytoplasmic \& Membranous positivity in Chromophobe RCC (score 5)

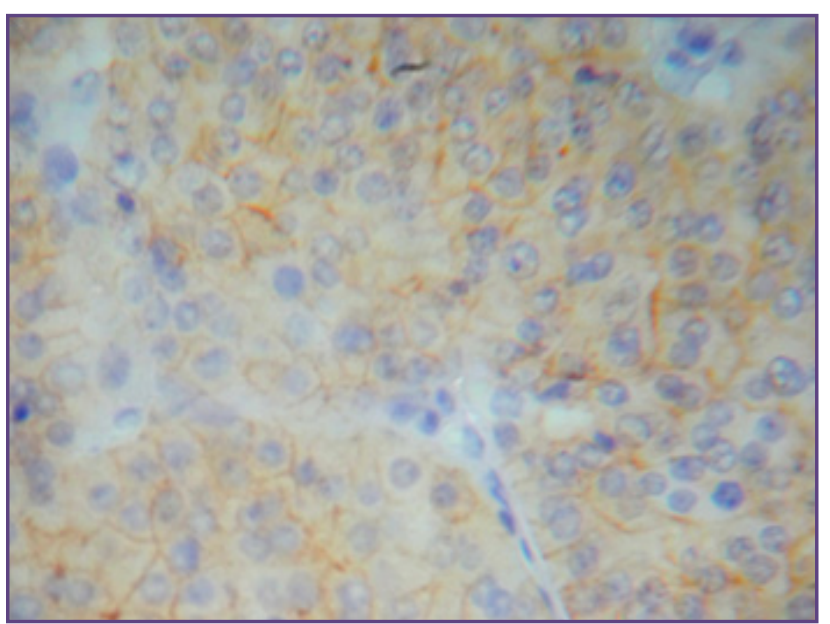

Fig. 4: CD117 (40x) Cytoplasmic \& Membranous positivity in Papillary RCC (score 5).

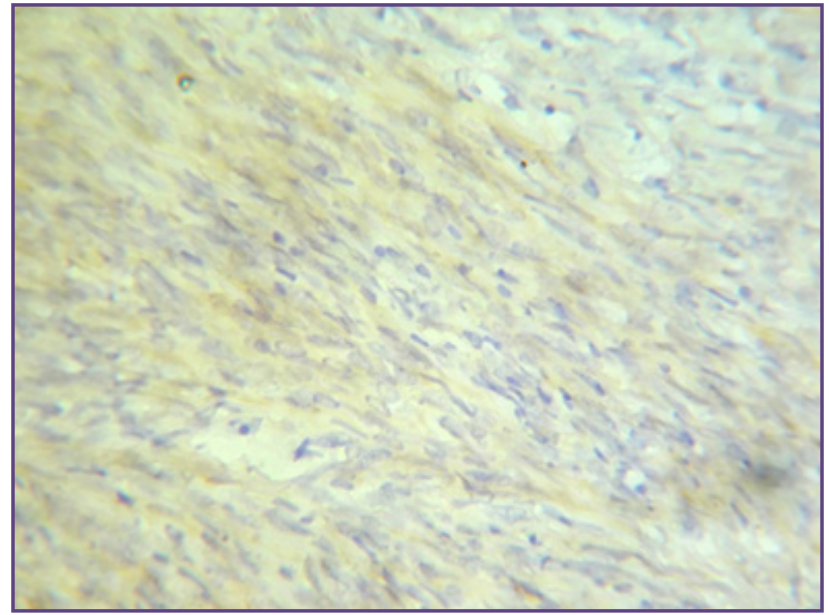

Fig. 5: CD 117- Focal cytoplasmic positivity inSarcomatoid RCC (score 2). 


\section{Discussion}

The current study included a total of 48 cases. Most patients $(44.2 \%)$ belonged to the age group of $50-70$ yrs. The finding is similar to a study reported by Atif Ali Hashmi et al on 50 cases of RCC that showed a mean age of 56.3. Another study reported by Ahmed EA and Youssif ME on 49 cases of RCC that showed age of the patients ranged from 20 to 80 years with a mean age of 53.14 and a median of $50 y$ rs.

In the present study, the male to female ratio was $3: 1$, showing a definite male preponderance. This was true with a study conducted by Ahmed Khalil Ibrahim on 44 cases of RCC which showed a similar male to female ratio of $3: 1$. Most other studies by DeCastro and McKiernon, (2008); Woldrich et al, (2008) and Carrizosa and Godley, (2009) showed a male to female ratio of 3:2.

At least one of the classic symptoms of renal cell carcinoma ie., palpable mass, hematuria or abdominal pain was present in 25 (52\% of the patients) cases. Incidentally detected patients constitute around 23 (48\%) cases, these included patients presenting with nonspecific symptoms for whom abdominal imaging led to the diagnosis of RCC. A study conducted by W.Y. Siow et al, on 165 cases of RCC, showed $39 \%$ of incidentally detected RCC cases

Out of the 48 specimens received, 37 were that of radical nephrectomy (77.1\%) and the remaining 11 (22.9\%) were of partial nephrectomy. The finding was similar in a study by Hung-Jui Tan et al on 7138 patients treated surgically for early stage renal cancer, they identified $5213(73.0 \%)$ cases of radical nephrectomy and, 1925 (27.0\%) of partial nephrectomy cases. Another study by R. Houston Thompson et al on 1,159 patients showed, 873 (75\%) cases of radical nephrectomy and and $286(25 \%)$ of partial nephrectomy cases

On gross examination most of the tumors were of the size $<7 \mathrm{~cm}$, that is 32 out of 48 patients $(66.67 \%)$ belonged to T1 group. Rest of the 16 cases had tumor size $>7 \mathrm{~cm}$. The findings were similar to another study conducted by Atif Ali Hashmi et al on 50 RCC cases of which, 17(34\%) were $<5 \mathrm{~cm}, 26$ cases $(52 \%)$ were between $5-10 \mathrm{~cm}$ and 7 cases (14\%) were of $>10 \mathrm{~cm}$.

In the present study, the most common type of RCC was Clear cell type, with variable cytoplasmic features ranging from clear cell in 30 cases $(62.5 \%)$ to mixed clear and oncocytic morphology in 2 cases. Five cases of Clear cell RCC showed sarcomatoid change in the form of pleomorphic sarcomatous areas constituting $10.42 \%$ of cases. Apart from these, Clear cells with Papillary areas were seen in 1 case. Papillary RCC and Chromophobe
RCC constituted around 6.25\% (3 cases) each. Other less common types of RCC includes, Unclassified RCC $4.17 \%$ ( 2 cases), Multilocular cystic RCC - 2.08\% ( 1 case), and Mucinous Tubular and Spindle Cell RCC - 2.08\% ( 1 case). Of the 5 cases with tumor size more than $10 \mathrm{~cm}$, all 5 showed clear cell histology with 2 showing focal sarcomatoid areas. In a study by Ahmed EA et al, on 49 cases of RCC, 30 cases $(61.22 \%)$ had clear cell histology. Another study by PR Rekha et al on 40 cases of RCC, 28 cases $(70 \%)$ had clear cell histology

Of the 48 cases in the present study only 6 cases, that is 2 case of Papillary RCC and 3 case of Chromophobe RCC showed moderate intensity and diffuse positivity for CD 117. Papillary RCC showed both cytoplasmic and membrane positivity, while Chromophobe RCC showed mainly membranous positivity. 1 case of clear cell RCC with sarcomatoid areas showed weak cytoplasmic positivity. While all other cases were found negative for CD 117. The positive cases were scored according to the intensity and extend of positivity used by Miliaras, et al. As the number of positive cases were minimal in the present study, statistical analysis was not done. In a study conducted by Eman Abdelzaher Ahmed et al on 49 cases of RCCs, 14 cases (28.57\%) showed immunostaining for c-KIT. The immunoreactive cases were distributed as 4 cases of Clear cell RCC, 7 cases of Chromophobe RCC, and 3 cases of Collecting duct RCC. The immunoreactive cases of clear cell RCC showed cytoplasmic staining of weak or moderate intensity. The staining pattern was focal in two cases and diffuse in the other two cases. Seven cases of Chromophobe RCC showed diffuse membranous immunoreactivity for c-KIT. As regards PRCC, all the studied cases lacked c-KIT immunoreactivity. In the study by Miliaras et al, on 25 cases of RCC, 2 of 13 conventional RCCs, 2 of 7 papillary type RCCs, and 4 of 7 chromophobe type RCCs were CD117 positive (Table: 2).

\section{Conclusion}

During the study period from July 2013 to june 2014, 48 cases of Renal cell carcinoma were studied. Clear cell RCC was the common histological type. CD 117 showed diffuse membranous positivity in Chromophobe RCC. Since chromophobe RCC has a better prognosis compared to other variants, this study shows the diagnostic usefulness of CD117 as a phenotypic marker for the same. In this era of molecular pathology, we hope that our study would establish the basis for a new possible therapeutic modality in Renal cell carcinoma.

\section{Reference}

1. Rekha PR, Rajendiran S, Rao S, Shroff S, Joseph LD, Prathiba D. Histological reclassification, histochemical 
characterization and CD117 immunoexpression in RCC. J Urol. 2008 ;24(3):343-347

2. Siegel R, Naishadham D, and Jemal A. Cancer Statistics 2013; CA: A Cancer Journal for Clinicians. 2013 ; 63(1):1130

3. Kato M, Suzuki T, Suzuki Y, Terasawa Y, Sasano H, Arai Y. Natural History of small renal cell carcinoma: evaluation of growth rate, histological grade, cell proliferation and apoptosis. J Urol. 2004 ;172(3):863-6

4. Tsui KH, Shvarts O, Smith RB, Figlin R, de Kernion JB, Belldegrun A. Renal cell carcinoma: prognostic significance of incidentally detected tumors. J Urol. 2001 ;163(2):426-30.

5. Skinnider BF., Amin MB. An immunohistochemical approach to the differential diagnosis of renal tumors. Semin Diagn Pathol. 2005; 22 (1): 51-68.
6. Ahmed EA, and Youssif ME. Immunohistochemical Study of c-KIT (CD117) Expression in Renal Cell Carcinoma J Egyptian Nat. Cancer Inst. 2009 ; 21(2): 121-132.

7. Athar U, Gentile TC. Treatment options for metastatic renal cell carcinoma: a review. Can J Urol. 2008;15: 3954-3966.

8. 1st EAU-ICUD - International Consultation on Kidney Cancer Barcelona-2010

9. Liu L, Qian J, Singh H, Meiers I, Zhou X, Bostwick DG. Immunohistochemical Analysis of Chromophobe Renal Cell Carcinoma, Renal Oncocytoma, and Clear Cell Carcinoma An Optimal and Practical Panel for Differential Diagnosis. Arch Pathol Lab Med. 2007;131:1290-1297

10. Stec R, Grala B, Mączewski M, Bodnar L and Szczylik C. Chromophobe renal cell cancer - review of the literature and potential methods of treating metastatic disease. Journal of Experimental \& Clinical Cancer Research. 2009, 28:134

*Corresponding author:

Rasmi Unnikrishnan, Sreepadam, Sahakarana Nagar, Thrikkumaramkudam, Ayyanthole P.O, Thrissur, Kerala, India.

Phone: +91 9995396049

Email: drrasmisudheer@gmail.com

Financial or other Competing Interests: None. 